\section{Original Article}

\author{
Corresponding Author \\ Ibrahim Obeid \\ (D) https://orcid.org/0000-0002-5602-0508 \\ L'Institut de la Colonne Vertébrale, CHU \\ Pellegrin, 33076 Bordeaux, France \\ Email: ibrahim.obeid@gmail.com
}

Received: May 8, 2021

Revised: June 19, 2021

Accepted: August 3, 2021

See the commentary on "Obeid-Coronal Malalignment Classification Is Age Related and Independently Associated to Personal Reported Outcome Measurement Scores in the Nonfused Spine" via https://doi. org/10.14245/ns.2142648.324.

\footnotetext{
(c) (i) (2)

This is an Open Access article distributed under the terms of the Creative Commons Attribution Non-Commercial License (https://creativecommons.org/licenses/by-nc/4.0/) which permits unrestricted non-commercial use, distribution, and reproduction in any medium, provided the original work is properly cited.
}

Copyright $\odot 2021$ by the Korean Spinal Neurosurgery Society

\title{
Obeid-Coronal Malalignment Classification Is Age Related and Independently Associated to Personal Reported Outcome Measurement Scores in the Nonfused Spine
}

David Christopher Kieser ${ }^{1}$, Louis Boissiere ${ }^{2}$, Anouar Bourghli ${ }^{3}$, Kazunori Hayashi ${ }^{2}$, Derek Cawley ${ }^{2}$, Caglar Yilgor ${ }^{4}$, Ahmet Alanay ${ }^{4}$, Emre Acaroglu ${ }^{5}$, Frank Kleinstueck ${ }^{6}$, Javier Pizones ${ }^{6}$, Ferran Pellise ${ }^{7}$, Francisco Javier Sanchez Perez-Grueso ${ }^{8}$, Ibrahim Obeid $^{2}$; on behalf of the European Spine Study Group

\author{
${ }^{1}$ Department of Orthopaedic Surgery and Musculoskeletal Medicine, University of Otago, Christchurch School \\ of Medicine, Christchurch, New Zealand \\ ${ }^{2}$ L’Institut de la Colonne Vertébrale, CHU Pellegrin, Bordeaux, France \\ ${ }^{3}$ Orthopedic and Spinal Surgery Department, Kingdom Hospital, Riyadh, Saudi Arabia \\ ${ }^{4}$ Acibadem University School of Medicine, Istanbul, Turkey \\ ${ }^{5}$ Spine Surgery Unit, Ankara Acibadem ARTES Spine Center, Ankara, Turkey \\ ${ }^{6}$ Spine Center, Schulthess Klinik, Zurich, Switzerland \\ ${ }^{7}$ Spine Surgery Unit, Hospital Universitario Val Hebron, Barcelona, Spain \\ ${ }^{8}$ Spine Surgery Unit, Hospital Universitario La Paz, Madrid, Spain
}

Objective: To evaluate Obeid-coronal malalignment (O-CM) modifiers according to age, sagittal alignment, and patient-reported outcome measures (PROMs), in the mobile spine. Methods: Retrospective review of a prospective multicenter adult spinal deformity (ASD) database with 1,243 (402 nonoperative, 841 operative) patients with no prior fusion surgery. Patients were included if they were aged over 18 years and were affected by spinal deformity defined by one of: Cobb angle $\geq 20^{\circ}$, pelvic tilt $\geq 25^{\circ}$, sagittal vertical axis $\geq 5 \mathrm{~cm}$, thoracic kyphosis $\geq 60^{\circ}$. Patients were classified according to the O-CM classification and compared to coronally aligned patients. Multivariate analysis was performed on the relationship between PROMs and age, global tilt (GT) and coronal malalignment (CM).

Results: Four hundred forty-three patients had CM of more than $2 \mathrm{~cm}$ compared to 800 who did not. The distribution of these modifiers was correlated to age. After multivariate analysis, using age and GT as confounding factors, we found that before the age of 50 years, $2 \mathrm{~A} 1$ patients had worse sex life and greater satisfaction than patients without CM. After 50 years of age, patients with $\mathrm{CM}(1 \mathrm{~A} 1,1 \mathrm{~A} 2)$ had worse self-image and those with $2 \mathrm{~A} 2,2 \mathrm{~B}$ had worse self-image, satisfaction, and 36-item Short Form Health Survey physical function. Self-image was the consistent determinant of patients opting for surgery for all ages. Conclusion: $\mathrm{CM}$ distribution according to $\mathrm{O}-\mathrm{CM}$ modifiers is age dependent. A clear correlation between the coronal malalignment and PROMs exists when using the O-CM classification and in the mobile spine, this typically affects self-image and satisfaction. Thus, CM classified according to O-CM modifiers is correlated to PROMs and should be considered in ASD.

Keywords: Spine, Deformity, Scoliosis, Coronal malalignment 


\section{INTRODUCTION}

Adult spinal deformity (ASD) describes a complex array of spinal conditions causing spinal deformity. ${ }^{1}$ ASD is common with a reported prevalence of $32 \%$ of patients aged over 50 years and $68 \%$ of patients aged over 70 years. ${ }^{2}$ The degree of deformity typically correlates with the patient's disability (quality of life, QoL). ${ }^{3}$ The predominant reason for this disability is that the spinal deformity induces spinal imbalance that prevents the normal economic resting posture of the spine, increasing the physiological demands of the spine and perispinal musculature. The resulting disability has been shown to directly relate to the degree of spinal imbalance. ${ }^{4-7}$ It is now well recognized that an imbalanced spine severely affects patient's function and QoL. $2,4,7-10$ Due to its high incidence and severe effects, ASD is estimated to have the greatest global disease burden of all common longterm disorders, including arthritis, chronic lung disease, congestive heart failure, diabetes, and ischemic heart disease. ${ }^{11}$

The most well-recognized correlation between spinal imbalance and disability is that of sagittal imbalance. Glassman and colleagues were the first to study the effects of spinal sagittal imbalance on functional outcomes and found that an increase in sagittal imbalance worsened functional outcomes. ${ }^{4}$ This finding has been confirmed in a number of subsequent publications. ${ }^{5-7,9,10}$

More recently the effects of coronal malalignment (CM) have been reported, with new classification systems developed to further understand the effect of coronal deformities on pain, function, and QoL. CM reflects the lateral deviation of the trunk over the pelvis and can be represented by the $\mathrm{C} 7$ plume line, with substantial displacement from the midline of the pelvis considered to be more than $20 \mathrm{~mm}$. In a recent classification, proposed by Obeid and colleagues, the Obeid-CM (O-CM) classification, the authors incorporate specific modifiers for each curve type, dependent on the direction of the $\mathrm{CM}$ in relation to the main curve of the deformity. ${ }^{12}$ The classification defines a concave $\mathrm{CM}$ (type 1) as the coronal plumbline being on the ipsilateral side of the concavity of the curve, in contrast to a convex CM (type 2) where the plumbline is on the convex side of the curve. This is further subtyped with type $1 \mathrm{~A}$ having the main curve apex between $\mathrm{T} 12$ and L4 and type $1 \mathrm{~B}$ having its apex above T12. Type $1 \mathrm{~A} 1$ is flexible and type $1 \mathrm{~A} 2$ is rigid. Type $2 \mathrm{~A}$ has the apex of the main curve between T12 and L4, whereas type 2B has the apex below L4. Type 2A1 has a normal lumbosacral junction and type $2 \mathrm{~A} 2$ has a degenerative lumbosacral junction (Fig. 1). Patients with no CM are defined as a $\mathrm{C} 7$ plumb line being within $2 \mathrm{~cm}$ of the central sacral vertical line.

\begin{tabular}{|c|l|l|}
\hline \multicolumn{1}{|c|}{ Types } & \multicolumn{2}{|c|}{ Subtypes } \\
\hline $\begin{array}{c}\text { Main coronal } \\
\text { curve types }\end{array}$ & $\begin{array}{c}\text { First modifier: } \\
\text { the apex of the curve }\end{array}$ & $\begin{array}{c}\text { Second modifier: } \\
\text { flexibility of curve }\end{array}$ \\
\hline $\begin{array}{c}\text { Type 1 } \\
\text { Concave }\end{array}$ & $\begin{array}{c}\text { Type 1A } \\
\text { between T12 and L4 }\end{array}$ & $\begin{array}{c}\text { Type 1A1 } \\
\text { flexible }\end{array}$ \\
\cline { 3 - 3 } & $\begin{array}{c}\text { Type 1A2 } \\
\text { rigid }\end{array}$ \\
\cline { 2 - 3 } & $\begin{array}{c}\text { Type 1B } \\
\text { above T11-12 }\end{array}$ \\
\hline \multirow{2}{*}{$\begin{array}{c}\text { Type 2 } \\
\text { Convex }\end{array}$} & $\begin{array}{c}\text { Type 2A } \\
\text { between T12 and L4 }\end{array}$ & $\begin{array}{c}\text { Type 2A1 } \\
\text { flexible }\end{array}$ \\
\cline { 2 - 3 } & \multicolumn{2}{|c|}{$\begin{array}{c}\text { Type 2A2 } \\
\text { rigid }\end{array}$} \\
\cline { 2 - 3 } & $\begin{array}{c}\text { Type 2B } \\
\text { Lumbosacral junction: below L4-5 }\end{array}$ \\
\hline
\end{tabular}

Fig. 1. Obeid-coronal malalignment classification.

The purpose of this study was to evaluate O-CM modifiers according to age, sagittal alignment, $\mathrm{CM}$, and patient-reported outcome measures (PROMs), in the mobile spine.

\section{MATERIALS AND METHODS}

We performed a retrospective review of a prospective multicenter ASD database with 1,243 ASD patients with no prior spinal fusion surgery. Ethical approval was obtained from Centre Hospitalier Universitaire de Bordeaux (IRB number: CE-GP-201914). Patients were included if they were aged at least 18 years, had no prior spinal fusion, and were affected by spinal deformity defined by at least one of the following: Cobb angle $\geq 20^{\circ}$, pelvic tilt $\geq 25^{\circ}$, sagittal vertical axis $\geq 5 \mathrm{~cm}$, or thoracic kyphosis $\geq 60^{\circ}$.

All patients completed Numerical Rating Scale back and leg pain scores, Oswestry Disability Index (ODI), 36-item Short Form Health Survey (SF-36), and Scoliosis Research Society 22 (SRS-22) scores. All patients were radiologically assessed according to the O-CM classification as well as global tilt (GT). Patients were classified according to the 6 modifiers of the O-CM classification and were compared to coronally aligned patients. ${ }^{12} \mathrm{We}$ then subsequently compared those patients that decided to undergo deformity correction to those that did not in order to determine which reported PROMS affected this decision.

Univariate and multivariate analysis was performed on the relationship between PROMs and age, sagittal GT, and CM using IBM SPSS Statistics ver. 25.0 (IBM Co., Armonk, NY, USA). We used GT as our sagittal parameter to exclude the confounding effect of sagittal balance on PROMs. Cross-tabulation was generated and chi-square test was used to compare all the dis- 
tributions of the modifiers according to age. Impaired t-tests or analysis of variance were performed to compare all groups of $\mathrm{CM}$ and to assess differences in means for PROMs. For each distribution, we used linear regression analysis to analyze the correlation between health-related QoL scores with GT and age and to determine if GT and/or age were confounding factors. In this case, we carried out a multivariate analysis using an analysis of covariance (ANCOVA) test for the PROMs for which we had a p-value less than 0.05 to determine if statistical significance persisted when taking into account the effect of GT and/or age.

\section{RESULTS}

A total of 1,243 patients were included. The mean age was 52 years (range, 18-90 years). Four hundred forty-three patients had CM of more than $2 \mathrm{~cm}$ compared to 800 who did not and the age distribution of CM appeared bimodal (Fig. 2). Eight hundred forty-one patients were subsequently elected for operative intervention and 402 elected for nonoperative treatment.

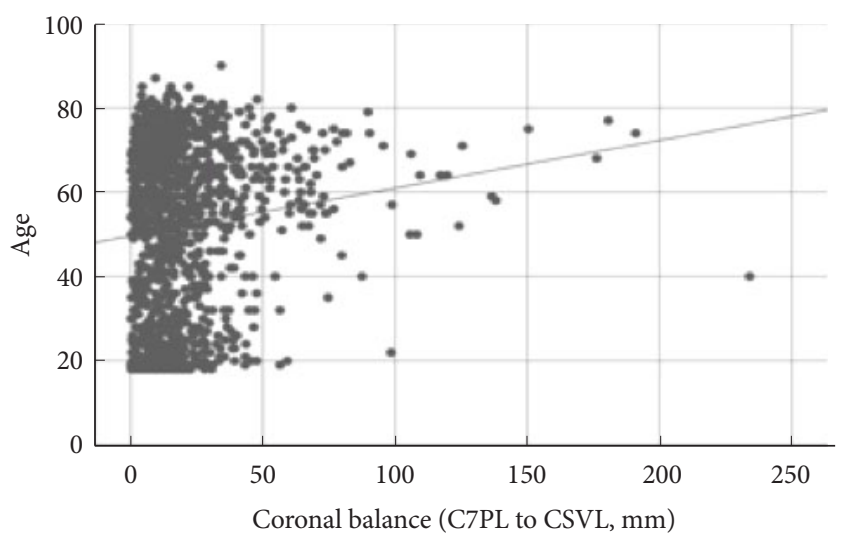

Fig. 2. Scatter plot showing the distribution of coronal alignment related to age. C7PL, C7 plumbline; CSVL, central sacral vertical line.

Table 1. The distribution of modifiers correlated to age

\begin{tabular}{lrc}
\hline Subtype & No. & Age (yr), mean (range) \\
\hline 1A1 & 56 & $65.0(19-90)$ \\
1A2 & 71 & $64.9(19-85)$ \\
1B & 23 & $43.6(18-75)$ \\
2A1 & 140 & $34.9(18-74)$ \\
2A2 & 113 & $63.7(28-82)$ \\
2B & 40 & $62.7(20-82)$ \\
No coronal malalignment & 800 & $50.9(18-87)$ \\
Total & 1,243 & $52.0(18-90)$ \\
\hline
\end{tabular}

The distribution of the modifiers was correlated to age; mean age was 35 years for $2 \mathrm{~A} 1$ patients, 44 years for $1 \mathrm{~B}$, and around 64 years for all other modifiers (Table 1 ).

When patients are grouped into an age of under 50 years compared to those older than 50 years, $76 \%$ of patients affected by $\mathrm{CM}$ who are under 50 years of age have subtype $2 \mathrm{~A} 1$. In contrast over the age of 50 years, the distribution of the O-CM varied more broadly (Table 2).

On univariate analysis, comparing the PROMs of those patients aged under 50 years with O-CM type 2A1 to those without coronal imbalance, the only statistically significant differences were a reduction in the ODI sex life ( 0.52 vs. $0.77, \mathrm{p}=0.033)$ and higher satisfaction ( 3.56 vs. $3.22, \mathrm{p}=0.015)$. No age or radiological confounders were identified and therefore no multivariate analysis performed in this group.

After 50 years of age, on univariate analysis, patients with coronal malalignment (O-CM classification 1A1/2) had a worse SRS-22 self-image ( 2.2 vs. $2.5, \mathrm{p}=0.000)$ and SF-36 physical function (33.3 vs. $35.2, \mathrm{p}=0.044$ ) than those with no CM. Similarly, patients with coronal malalignment $\mathrm{O}-\mathrm{CM}$ classification $2 \mathrm{~A} 2$ and $2 \mathrm{~B}$ had worse SRS-22 self-image (2.3 vs. $2.5, \mathrm{p}=0.000)$, SRS-22 satisfaction (2.8 vs. 3.2, $\mathrm{p}=0.002)$ and SF-36 physical function ( 32.9 vs. $35.2, \mathrm{p}=0.015)$ than those with no CM. In contrast, only those with O-CM classification $1 \mathrm{~A} 1 / 2$ had significantly greater GT (40.6 vs. 30.7, p=0.000). On multivariate analysis, accounting for age and GT as confounders in patients with O-CM classification 1A1/2, only the SRS-22 self-image remained significant (2.2 vs. $2.5, \mathrm{p}=0.014$ ).

When comparing those patients that subsequently elected for continued nonoperative care to those who undergo deformity correction, in those younger than 50 years, we found that the nonoperative cohort O-CM 2A1 initially presented with a worse ODI sex life ( 0.26 vs. $0.55, \mathrm{p}=0.026)$ than their coronally balan-

Table 2. The frequency of Obeid-coronal malalignment subgroups under and over the age of 50 years

\begin{tabular}{lcc}
\hline Subtype & Age $<50 \mathrm{yr}$ & Age $\geq 50 \mathrm{yr}$ \\
\hline $1 \mathrm{~A} 1$ & $5(1.0)$ & $51(6.7)$ \\
$1 \mathrm{~A} 2$ & $1(0.2)$ & $70(9.2)$ \\
1B & $13(2.7)$ & $10(1.3)$ \\
$2 \mathrm{~A} 1$ & $110(22.9)$ & $30(3.9)$ \\
$2 \mathrm{~A} 2$ & $9(1.9)$ & $104(13.6)$ \\
2B & $7(1.5)$ & $33(4.3)$ \\
No coronal malalignment & $335(69.8)$ & $465(60.9)$
\end{tabular}

Values are presented as number (\%). 
ced counterparts. All other PROMS and GT were similar. However, age was a confounding factor (ANCOVA, $p=0.104$ ), and on multivariate analysis no difference when accounting for age between the $2 \mathrm{~A} 1$ and coronal balanced patients ODI sex life was found. In contrast, those that subsequently elected for surgical correction had a worse SRS-22 self-image ( 2.5 vs. $2.8, \mathrm{p}=0.020)$ and better SRS-22 satisfaction (3.5 vs. 3.0, p=0.014) than their coronally balanced counterparts. The age and GT were again similar.

For those aged over 50 years, the nonoperative group with O-CM 1A1/2 had worse SF-36 physical function (36.0 vs. 40.0, $\mathrm{p}=0.039)$ than those who were coronally aligned. There was no significant difference in GT (35.0 vs. 27.1, p=0.066) and therefore no multivariate analysis was performed. For those with $\mathrm{O}-\mathrm{CM} 2 \mathrm{~A} 2$ and $2 \mathrm{~B}$, there were no identifiable differences in PROMS or GT between the coronally aligned and mal-aligned groups that elected for nonoperative care.

In contrast, those aged over 50 years that elected for operative intervention with O-CM 1A1/2 had worse SRS-22 self-image ( 2.0 vs. $2.3, \mathrm{p}=0.000)$ than those who were coronally aligned. They also had a greater GT (42.9 vs. $32.1, \mathrm{p}=0.000)$. On multivariate analysis, accounting for age and GT, the SRS-22 self-image remained lower for those with $\mathrm{O}-\mathrm{CM} 1 \mathrm{~A} 1 / 2$ (2.0 vs. 2.3, $\mathrm{p}=0.007)$. Patients with O-CM $2 \mathrm{~A} 2$ and $2 \mathrm{~B}$ had worse SRS-22 self-image (2.1 vs. $2.3, \mathrm{p}=0.014)$ and SRS-22 satisfaction (2.7 vs. 3.1, $\mathrm{p}=0.004$ ) than those who were coronally aligned. When accounting for age and GT the trend remained with a worse SRS-22 self-image ( 2.1 vs. $2.3, \mathrm{p}=0.031)$ and SRS-22 satisfaction $(2.7$ vs. $3.1, \mathrm{p}=0.003)$ in the $\mathrm{O}-\mathrm{CM} 2 \mathrm{~A} 2$ and $2 \mathrm{~B}$ group. There was no other statistically significant difference between the groups in relation to PROMS or GT.

\section{DISCUSSION}

While sagittal imbalance is well recognized as correlating to patient outcomes, there is inconsistent evidence about the effect of coronal deformities on patient pain, function, and QoL., ${ }^{4,13,14}$ However, with a number of new classifications recently proposed there is a resurgence of interest in $\mathrm{CM} \cdot{ }^{12,14-17}$ In a recent study published by Plais et al., ${ }^{18}$ the authors assessed the effect of CM in relation to the Qui classification in mobile spines and found an age-dependent variance in the curve type and a relationship between the degree of CM greater than $3 \mathrm{~cm}$ to functional outcomes.

In the present study, we assessed similar variables, but utilized the $\mathrm{O}-\mathrm{CM}$ classification. We found that a $\mathrm{CM}$ of $2 \mathrm{~cm}$ or great- er affected PROMs, suggesting that the O-CM classification is more sensitive than other published classifications for detecting the effects of CM on patient outcomes. We found a similar age variance to the curve type reported by Plais et al. ${ }^{18}$ with most patients under the age of 50 years being affected by O-CM subtype $2 \mathrm{~A} 1$, and those older than 50 years being more evenly affected by different subtypes. We chose 50 years because our dataset suggested a bimodal distribution with 50 years being between the peaks (Fig. 1). In addition, the article by Fujishiro et al. ${ }^{19,20}$ used an age of 40 years as a cutoff because the authors noted patients typically start to become symptomatic after this age. Furthermore, Yilgor et al. ${ }^{21}$ used age 60 years for the Global Alignment and Proportion score because most complications were thought to present around this age. In our study, we were assessing the deformity itself and age 50 years was therefore deemed appropriate.

We also identified that coronal imbalance variably affects specific outcomes according to the age and curve type. Patients aged under 50 years with CM have a worse sex life, but higher satisfaction. In contrast, those older than 50 years with O-CM $1 \mathrm{~A} 1$ or $1 \mathrm{~A} 2$ had worse self-image and those with $2 \mathrm{~A} 1$ and $2 \mathrm{~B}$ had worse self-image, satisfaction, and physical function.

In addition, when assessing which patients subsequently elected for spinal deformity correction, irrelevant of age, self-image was the only consistent factor. Unexpectedly, patient satisfaction was in fact higher in those aged less than 50 years who subsequently elected for surgery in contrast to those older than 50 whose satisfaction was lower. This suggests that self-image is the predominant determinant of surgical intervention in younger patients, whereas satisfaction and self-image drive decision making in older patients.

Furthermore, the fact that only specific PROMs, notably selfimage and satisfaction, rather than overall QoL and function were altered suggests that surgeons contemplating surgical intervention for CM should recognize these factors as predominant determinants of patient decision making and council them accordingly.

This study offers several advantages to previous literature: notably its large sample size, its multivariate analysis, the consideration of different curve types to subanalyse CM, and the effects on specific PROMS. However, despite these advantages, this study has a number of limitations. Firstly, for each age bracket, we analyzed the most common deformity patterns and therefore our results cannot be translated to more rare deformity subtypes. Secondly, we used GT as a composite of sagittal imbalance and therefore other parameters of sagittal deformity can- 
not be excluded as confounders. Lastly, we have not assessed the effect of surgical intervention on improving patient outcomes.

Despite these limitations, this study shows that CM clearly affects PROMs and the disparity with the previous literature is due probably to the fact that only general types of CM were described, bypassing the different subtypes and eventually confusing them. In addition, the current study has shown that the CM pattern is age related and that the age distribution is not homogenous which we believe needs to be understood for the patient's best management and eventual operative planning if surgery is indicated.

\section{CONCLUSION}

$\mathrm{CM}$ distribution according to $\mathrm{O}-\mathrm{CM}$ modifiers is age dependent. Despite previous reports failing to correlate CM with PROMs, our study shows that when each modifier is considered a clear correlation exists. In the nonfused spine, an independent correlation between CM and PROMs affecting specifically self-image and satisfaction was shown. Thus, CM classified according to O-CM modifiers is correlated to PROMs and should be considered in ASD.

\section{CONFLICT OF INTEREST}

The authors have nothing to disclose.

\section{ACKNOWLEDGMENTS}

We appreciate Glynny Kieser for her grammatical and editorial input.

\section{REFERENCES}

1. Good CR, Auerbach JD, O'Leary PT, et al. Adult spine deformity. Curr Rev Musculoskelet Med 2011;4:159-67.

2. Schwab F, Dubey A, Gamez L, et al. Adult scoliosis: prevalence, SF-36, and nutritional parameters in an elderly volunteer population. Spine (Phila Pa 1976) 2005;30:1082-5.

3. Acaroglu E, Yavuz AC, Guler UO, et al. A decision analysis to identify the ideal treatment for adult spinal deformity: is surgery better than non-surgical treatment in improving health-related quality of life and decreasing the disease burden? Eur Spine J 2016;25:2390-400.

4. Glassman SD, Bridwell K, Dimar JR, et al. The impact of positive sagittal balance in adult spinal deformity. Spine (Phi- la Pa 1976) 2005;30:2024-9.

5. Lafage V, Schwab F, Patel A, et al. Pelvic tilt and truncal inclination: two key radiographic parameters in the setting of adults with spinal deformity. Spine (Phila Pa 1976) 2009;34: E599-606.

6. Schwab F, Patel A, Ungar B, et al. Adult spinal deformitypostoperative standing imbalance: how much can you tolerate? An overview of key parameters in assessing alignment and planning corrective surgery. Spine (Phila Pa 1976) 2010; 35:2224-31.

7. Schwab FJ, Blondel B, Bess S, et al. Radiographical spinopelvic parameters and disability in the setting of adult spinal deformity: a prospective multicenter analysis. Spine (Phila Pa 1976) 2013;38:E803-12.

8. Schwab F, Dubey A, Pagala M, et al. Adult scoliosis: a health assessment analysis by SF-36. Spine (Phila Pa 1976) 2003;28: 602-6.

9. Cawley DT, Larrieu D, Fujishiro T, et al. Back and leg score: an appropriate outcome measure for adult spinal deformity surgery. Spine J 2017;17(10 Suppl):S238.

10. Kieser D, Boissière L, Ghailane $S$, et al. Adult spinal deformity (ASD) surgery: Understanding the true impact on specific activities of daily living. Leb Med J 2018;66:535-40.

11. Pellise F, Vila-Casademunt A, Ferrer M, et al. Impact on health related quality of life of adult spinal deformity (ASD) compared with other chronic conditions. Eur Spine J 2015; 24:3-11.

12. Obeid I, Berjano P, Lamartina C, et al. Classification of coronal imbalance in adult scoliosis and spine deformity: a treatment-oriented guideline. Eur Spine J 2019;28:94-113.

13. Deviren V, Berven S, Kleinstueck F, et al. Predictors of flexibility and pain patterns in thoracolumbar and lumbar idiopathic scoliosis. Spine (Phila Pa 1976) 2002;27:2346-9.

14. Schwab F, Ungar B, Blondel B, et al. Scoliosis Research Society-Schwab adult spinal deformity classification. Spine (Phila Pa 1976) 2012;37:1077-82.

15. Bao H, Yan P, Qiu Y, et al. Coronal imbalance in degenerative lumbar scoliosis: prevalence and influence on surgical decision-making for spine osteotomy. Bone Joint J 2016;98B:1227-33.

16. Buell TJ, Smith JS, Shaffrey CI, et al. Multicenter assessment of surgical outcomes in adult spinal deformity patients with severe global coronal malalignment: determination of target coronal realignment threshold. J Neurosurg Spine 2020;4:114.

17. Lau D, Haddad AF, Deviren V, et al. Asymmetrical pedicle 
subtraction osteotomy for correction of concurrent sagittalcoronal imbalance in adult spinal deformity: a comparative analysis. J Neurosurg Spine 2020 Aug 7:1-8. https://doi.org/ 10.3171/2020.5.SPINE20445. [Epub].

18. Plais N, Bao H, Lafage R, et al. The clinical impact of global coronal malalignment is underestimated in adult patients with thoracolumbar scoliosis. Spine Deform 2020;8:105-13.

19. Fujishiro T, Boissière L, Cawley DT, et al. Adult spinal deformity surgical decision-making score: Part 1: development and validation of a scoring system to guide the selection of treatment modalities for patients below 40 years with adult spinal deformity. Eur Spine J 2019;28:1652-60.

20. Fujishiro T, Boissière L, Cawley DT, et al. Adult spinal deformity surgical decision-making score. Part 2: development and validation of a scoring system to guide the selection of treatment modalities for patients above 40 years with adult spinal deformity. Eur Spine J 2020;29:45-53.

21. Yilgor C, Sogunmez N, Boissiere L, et al. Global Alignment and Proportion (GAP) score: Development and validation of a new method of analyzing spinopelvic alignment to predict mechanical complications after adult spinal deformity surgery. J Bone Joint Surg Am 2017;99:1661-72. 